\title{
A Hierarchical Self-Organising Fuzzy Logic-Based On-Line Advisor for the Management of Cardiac Septic Patients
}

\author{
M. Mahfouf*, O. King*, M. Denaï** \\ J.J. Ross***, Qing Lu**** \\ * Dept of Automatic Control and Systems Eng, University of Sheffield S1 3JD \\ UK (Tel: +44 114 2225607; e-mail: m.mahfouf@shef.ac.uk). \\ ** School of Science \& Eng, Teesside University, Middlesbrough, TS1 3BA, UK (e-mail: \\ m.denai@tees.ac.uk) \\ *** Dept of Anaesthesia, Northern General Hospital, Sheffield S5 7AU, UK (e-mail: j.j.ross@shef.ac.uk) \\ $\star \star \star \star$ Research Centre for Learning Sciences, Southeast University, Nanjing, 210096, China
}

Abstract: An on-line intelligent advisory system (IAS) for the management of septic patients emerging from cardiopulmonary bypass (CPB) has been developed and evaluated on a comprehensive physiological model which incorporates sepsis-induced capillary leak and the hemodynamic response to Intensive Care Unit (ICU)-like therapeutic interventions. The IAS is composed of the expert's decision-making model which performs diagnosis and designates the appropriate therapeutic actions based on the patient's hemodynamic status and a multi-inputs multi-outputs self-organising fuzzy logic controller (SOFLC) for adjusting the infusion rates of the selected drugs to maintain the hemodynamics variables at the specified target values. Simulated patient scenarios reproducing post-CPB hemodynamic abnormalities were developed with the expert anesthetist to test the IAS ability to advice for the appropriate therapeutic interventions whilst controlling individual drugs infusion rates to maintain the hemodynamic parameters at the prescribed targets values.

\section{INTRODUCTION}

Septic shock is the most severe pathophysiological condition on the continuum of the body response to infection. Patients with sepsis show evidence of infection and clinical manifestations of inflammation. Severe sepsis is the development of hypoperfusion with organ dysfunction in a septic patient, and septic shock is hypoperfusion and persistent hypotension in patients with sepsis. Most studies show that mortality rates lie between $40 \%$ and $60 \%$ for most types of infections leading to septic shock (Dellinger, 2003). Major surgery carries a risk of exogenous infection through exposed surfaces and intravenous lines, but studies have shown that $90 \%$ of the organisms implicated in septic shock come from the body's internal flora. Alterations in peripheral vascular tone and cardiac function contribute to the cardiovascular manifestations of septic shock (Bridges and Dukes, 2005; Parillo, 1989). Heart rate and cardiac output commonly remain normal or increase. Systemic vascular resistance (SVR) is low because arteriolar tone is decreased. Despite the increase in cardiac output (CO), tissue hypoperfusion is characterised by a raised blood lactate concentration, with oxygen extraction at less than maximum (Astiz and Rackow, 1998). Experimental studies show a loss of capillary cross section, and unusual microvascular effects such as the shunting of blood past organs (Groscott and Mythen, 2001). Hypovolemia is a major factor that contributes to the initial hemodynamic instability in septic shock. Patients have characteristically a low cardiac filling pressure and a low cardiac output in the early stages of shock. Their cardiac output only increases after fluid resuscitation. Hypovolemia is caused by a decreased venous tone, which increases the capacity of the veins. Much of the circulating volume is also lost through a capillary leak, as sepsis increases the microvascular permeability. Fluid is lost in the "third space", and reabsorbed if and when the underlying infection is treated. Coronary perfusion and myocardial oxygen metabolism remain normal during septic shock. However, some myocardial dysfunction is seen in most patients with septic shock, especially in post heart surgery patient population.

Clinicians must continuously monitor the condition of the patient using a variety of invasive and non-invasive measures at the same time as devising and executing an appropriate treatment plan. After identifying and reversing the cause of infection with antibiotics, the main method of managing sepsis/septic shock is with intravenous fluid and drug infusions. The clinician typically has a large number of drugs to choose from to treat each manifestation of sepsis. The infusion rates are set in order to maintain patient stability and drive measured parameters towards clinical endpoints. The skills and thought processes needed for this have been likened as much to an art than a science. This takes place against the hectic backdrop of an ICU during open-heart surgery where goals may change at the surgeons request or in response to the patient's condition.

A computerised decision support/advisory system can prove particularly useful in this challenging control environment.

In this research study an intelligent advisory system (IAS) integrating expert knowledge and self-organising fuzzy logic control theory is developed for automating the decisionmaking strategies in septic patients emerging from cardiac surgery and treated with cardiopulmonary bypass (CPB). The 
IAS is comprehensively tested on a physiological model describing the human cardiovascular mechanics and fluid/proteins exchange in the capillaries. The physiological model hence developed can reasonably reproduce the major hemodynamic abnormalities observed in cardiac septic patients and successfully predicts the response to different therapeutic interventions such us fluid, inotropic drugs, vasoconstrictor and vasodilators.

The rest of the paper is organized as follows. Section 2 focuses on the description of the physiological model. Section 3 deals specifically with the description and implementation aspects of the IAS. Section 4 presents a simulation study to evaluate the SOFLC-based hemodynamic controller. The evaluation of the IAS under different model scenarios replicating common conditions observed in postCPB septic patients is presented in section 5. Finally, conclusions relating to this research contribution are summarised in Section 6 of the paper.

\section{PHYSIOLOGICAL MODELING OF THE CARDIAC FUNCTION IN SEPTIC PATIENTS}

The objective here was to develop a physiological model that is able to reproduce the key hemodynamic variables of the cardiovascular function during and after CPB. The physiological model depicted in Fig. 1 combines ideas and equations from two previous models of the cardiovascular hemodynamics (Randall, 1986; Mason, 1989) and fluid exchange in the microcirculation (Xie, 1995).

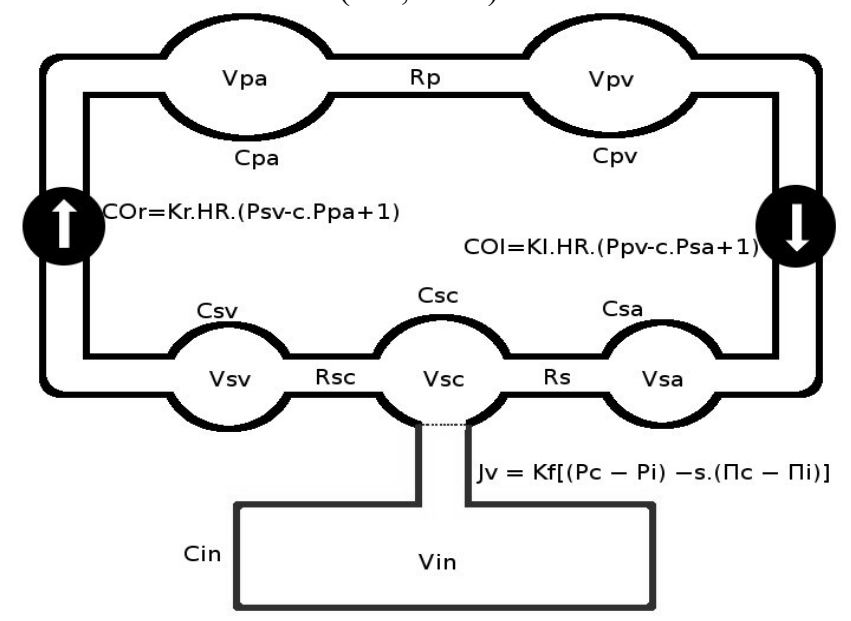

Fig. 1. Schematic diagram of the physiological model. Subscript 'p' denotes pulmonary, 's' systemic, 'a' arterial and 'v' venous (Randall, 1986; Mason, 1989; Xie, 1995)

The flow through the left and right heart, i.e. the cardiac output, is described by the following empirically derived equations:

$C O_{l}=K_{l} \cdot H R \cdot\left(P_{p v}-c \cdot P_{s a}+1\right)$

$C O_{r}=K_{r} \cdot H R \cdot\left(P_{s v}-c \cdot P_{p a}+1\right)$

Where $K_{l}$ and $K_{r}$ are the contractilities of the left and right ventricle, $P$ is the pressure and $H R$ is the heart rate. Subscripts $p$ and $s$ denote pulmonary and systemic, $v$ and $a$ denote veins and arteries. The compliance and resistance parameters were taken from (Xie, 1995; Gyenge et al., 1999).
The baroreflex effect on the mean arterial pressure (MAP) to the heart rate (HR) and systemic vascular resistance (SVR) is represented by a piecewise linear relationship with transport delay. Drug effects were incorporated in a similar way. A piecewise linear relationship with transport delay was assumed to exist between drug infusion and its effect on SVR and ventricles' contractilities $\left(K_{l}\right.$, and $\left.K_{r}\right)$. The model was able to simulate the effects of vasodilators, vasopressors and inotropes. New drugs can be trivially added to this model by defining the infusion rate/effect relationship. In addition, fluid can be added to the circulation to simulate fluid resuscitation in response to hypovolemia and/or low blood pressure. A constant urine output was also included in the model.

The Cardiovascular System (CVS) model is extended with an additional compartment describing the transport and distribution of fluid and plasma proteins between the circulation and the interstitial space (Xie, 1995; Gyenge et al., 1999). In the microcirculation, fluid and protein balance is maintained by hydrostatic and oncotic pressures existing on either sides of the capillary barrier according to Starling's equation which described the rate of fluid filtration from the circulation to the interstitial space (Gyenge et al., 1999; Klabunde, 2004).

$J_{i}=K_{f}\left[\left(P_{c}-P_{i}\right)-\sigma\left(\Pi_{c}-\Pi_{i}\right)\right]$

Where $J_{i}$ represents the net fluid movement between the compartments, $K_{f}$ is the fluid filtration coefficient, $P_{c}$ and $P_{i}$ represent the capillary and interstitial hydrostatic pressures respectively, $\Pi_{c}$ and $\Pi_{i}$ represent the capillary and interstitial oncotic pressures respectively, $\sigma$ denotes the reflection coefficient, a measure of the permeability of the capillaries to the proteins responsible for generating the oncotic pressure.

Different pathophysiological conditions can be simulated by changing the model parameters. Reducing the reflection coefficient $\sigma$ by $50 \%$ increases the permeability of the capillaries, causing a capillary leak of over $500 \mathrm{ml}$. This, in turn, causes a reduction in MAP and $\mathrm{CO}$, mimicking the initial effects of sepsis induced capillary leak. However, in many real patients the reflexes will raise the HR and heart contractility, hence maintaining $\mathrm{CO}$.

Increasing the SVR will cause an increase in MAP. However, the increased afterload will reduce the CO. SVR may change due to drugs or reflex responses. The onset of severe sepsis is accompanied by an impairment of the autonomous nervous system leading to a compromised baroreflex function with a deregulation in the sympathetic and parasympathetic activities. Therefore, to better illustrate the interaction between drugs and the physiological model, the baroreflex function has been disabled in this simulation study.

The model parameters have been tuned (optimized) using real clinical data (Hamada et al., 2004).

\section{DESIGN METHODOLOGY OF THE IAS FOR CARDIAC SEPTIC PATIENTS}

The overall structure of the IAS depicted in Fig. 2 integrates the fuzzy decision rule-base which performs diagnosis based on the hemodynamic parameters and selects the appropriate 
therapy and a set of SOFLC to adjust the infusion rates of drugs. Patient model

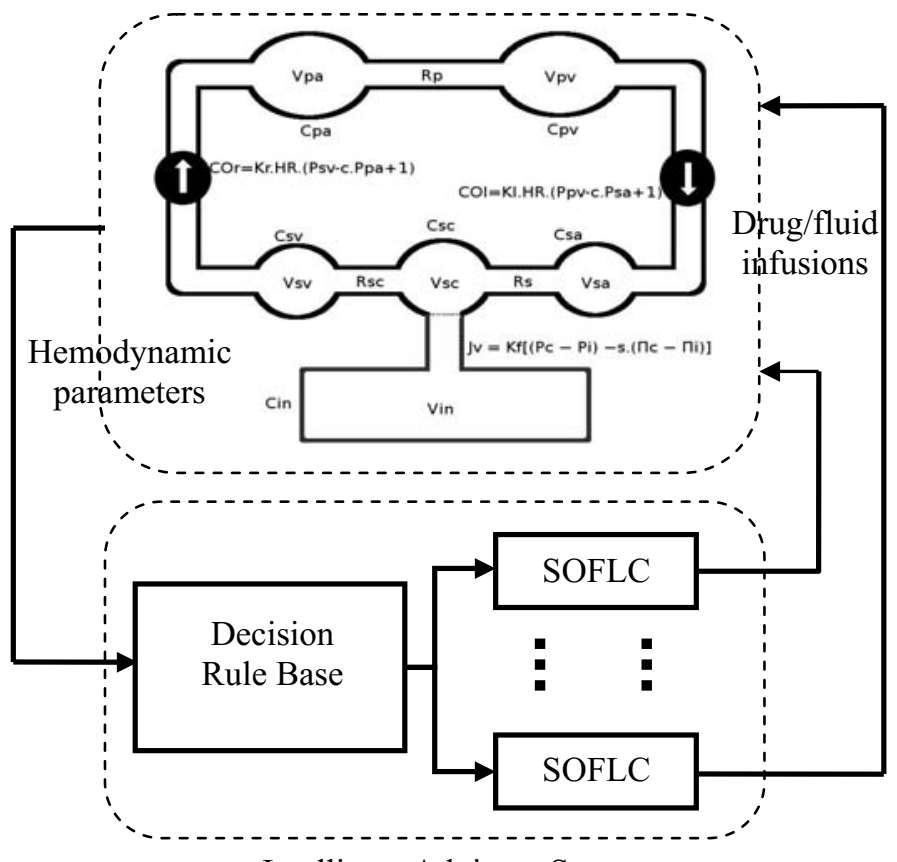

Intelligent Advisory System

Fig. 2: Schematic diagram of the IAS with the patient's model

\subsection{Decision Rule Base}

The authors have elaborated, in collaboration with an expert anesthetist (Ross et al., 2006), a conceptual model of the customary clinical decision pathway that a senior anesthetist with substantial experience uses to support post-CPB patients. The model refers to five components of the CVS mainly the systolic blood pressure (SBP), central venous pressure (CVP), systemic vascular resistance (SVR), cardiac output (CO) and heart rate (HR). The decision levels associated with these components are prioritized as shown in Fig. 3.

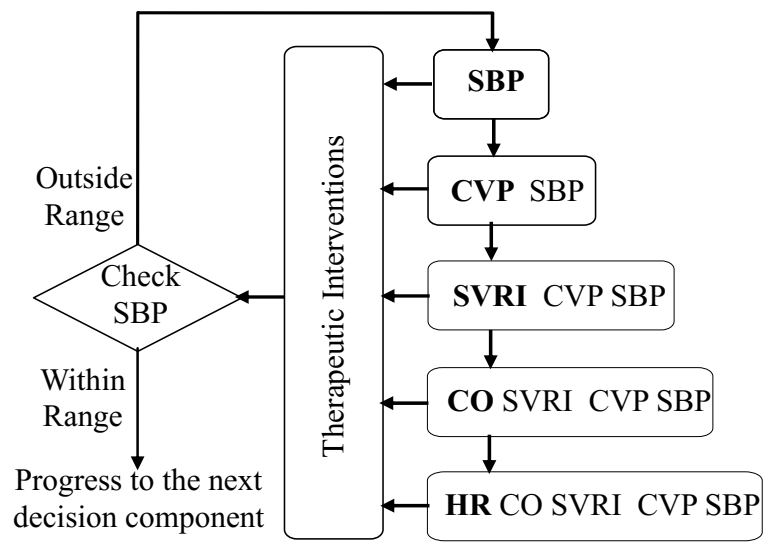

Fig. 3. Synopsis of the decision-making model

However, as it is a dynamic environment, and treatments may have effects on several components, it is necessary to continuously revisit those previously treated.

The Decision Rule Base implementing the decision model of Fig. 3 were encoded as a set of fuzzy linguistic rules as it seemed the most natural translation between the expert's knowledge, the inputs and a crisp decision. Defining acceptable bounds for each fuzzy set presented some difficulty as these may change under different circumstances. Additionally, values such as the CVP and the HR may not have ideal values as such; they are relative to the pre-surgery values for each patient. Since information like this cannot be encoded into a one size fits all rule-base, it is important that these can be modified by the user in any implementation. An example of fuzzy partition is shown in Fig. 4 for the MAP.

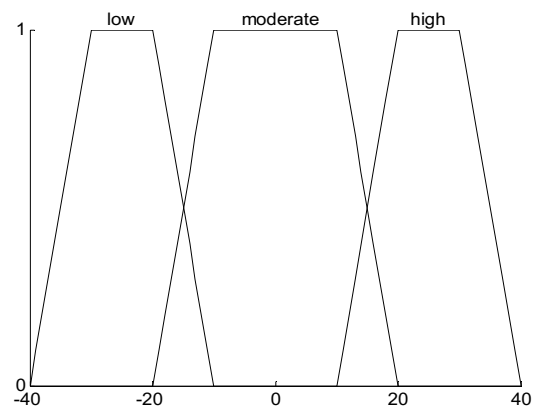

Fig. 4. Membership functions definition for the MAP

\subsection{Design of the Multiple-Drug SOFLC Controller}

Self-organising fuzzy logic control (SOFLC) works on the principle of continuous on-line performance evaluation, and if necessary, rule modification of an existing fuzzy logic controller, as proposed by (Procyk and Mamdani, 1979).

Usually, the error and the change in error are taken as inputs, and the output is the required change in the control action - PI type controller. The basic configuration of a SOFLC is shown in Fig. 5.

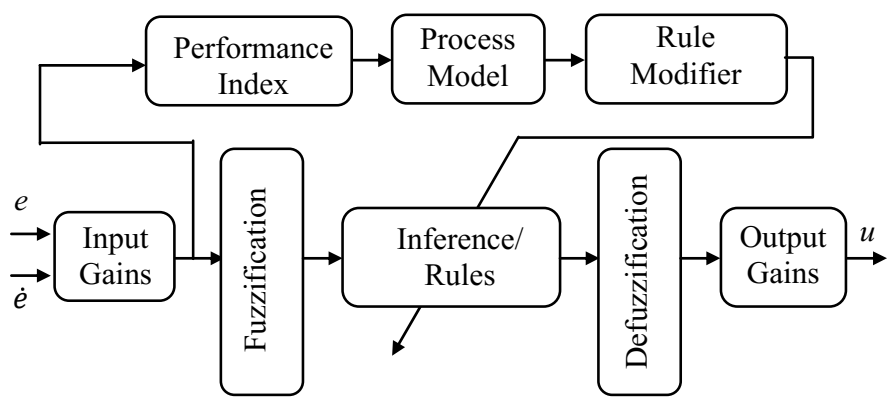

Fig. 5. Basic configuration of SOFLC

Where $e$ and $\dot{e}$ denote the error (difference between the hemodynamic variable and its target value) and error change respectively and $u$ in the controller output representing the infusion rate of the drug to be administered under these conditions. The input and output gains were adjusted through trial and error. The performance index describing the desired response is usually in the form of rules which indicate the correction needed to the control output and hence the fuzzy rule-base. This correction is applied to the past control output most responsible for the current undesirable state, hence some knowledge of plant delays is necessary. In equation form, the adjustment rule is as follows:

$F(i, j)_{n-d}=F(i, j)_{n-d}+P I(i, j)_{n-d}$ 
Where $F$ is the current rule base, $i$ and $j$ are the indices which correspond to the current error and change of error, $P I$ is the performance index, $n$ is the current sample time and $d$ is the delay.

A third-order polynomial filter with a window of 20 samples was used to improve the performance of the SOFLC under noisy conditions. Polynomial filtering uses a least-squares fitting of a polynomial function to the noisy signal over a fixed window. The general form of this estimated function is:

$p(x)=\sum_{i=1}^{m} c_{k} \phi_{k}(x)$

Where $\phi(x)$ is the $k^{\text {th }}$ order polynomial function, the factors $c_{1}$ to $c_{m}$ can be found by solving the following equation:

$\frac{\partial E}{\partial c_{j}}=\sum_{k=1}^{n} 2\left[\sum_{i=1}^{m} c_{i} \phi_{i}(x(k))-y(k)\right] \phi_{j}(x(k))=0$

Where $E=\sum_{k=1}^{n}\left[\sum_{i=1}^{m} c_{i} \varphi_{i}(x(k))-y(k)\right]^{2}$

A window of $n$ samples of noisy data $(y(k), k=t-n+$ $1, t-n+2, \cdots, t)$ is used to calculate the current smoothed data point $\hat{y}(t)=\sum_{i=1}^{m} c_{i} \phi_{i}(x(t))$ using the previously calculated factors. The smoothed signal $\hat{y}(t)$ and its derivative $\hat{y}(t)-\hat{y}(t-1)$ are used in the control scheme.

Vasoactive and inotropic drugs have direct effects on their sites of action; however there may also be indirect effects and secondary effects on other parameters. For instance, a pure vasoconstrictor will not only raise the systemic blood pressure, it will affect the pressure immediately before and after the heart and change the cardiac output. In multivariable control such interaction can have two undesirable effects:

- Cross-coupling between main loops may lead to large output fluctuations especially in the midfrequency range;

- It may make controller tuning more difficult.

Relative gain array (RGA) (Bristol, 1966) provides a measure of control interaction and a method of reducing its effects.

The RGA for an n-input n-output system can be defined as follows:

$\Lambda=\left[\begin{array}{cccc}\lambda_{11} & \lambda_{12} & \cdots & \lambda_{1 \mathrm{n}} \\ \lambda_{21} & & & \lambda_{2 \mathrm{n}} \\ \vdots & & & \vdots \\ \lambda_{n 1} & \lambda_{n 2} & \cdots & \lambda_{n n}\end{array}\right]$

Where

$\lambda_{i j}=\frac{\text { Open loop gain between } y_{i} \& u_{j}}{\text { Closed loop gain between } y_{i} \& u_{j}}$

For a two-input/two-output system, $\lambda_{11}$ can be defined as follows

$\lambda_{11}=\frac{1}{1-\frac{K_{12} \cdot K_{21}}{K_{11} \cdot K_{22}}}$

Where

$$
K_{11}=\frac{\Delta y_{1}}{\Delta u_{1}} \text { with respect to } u_{2} K_{12}=\frac{\Delta y_{1}}{\Delta u_{2}} \text { with respect to } u_{2}
$$

$$
K_{21}=\frac{\Delta y_{2}}{\Delta u_{1}} \text { with respect to } u_{2} K_{12}=\frac{\Delta y_{2}}{\Delta u_{2}} \text { with respect to } u_{2}
$$

The other terms of $\Lambda$ can be easily calculated as all rows and column sum to 1 . The modified inputs $\bar{u}$ can then be defined as $\bar{u}=\Lambda u$. RGA provides a powerful way of measuring and compensating for loop interactions. However, on its own it does have significant weaknesses. This method will not provide perfect decoupling as it assumes a linearised model around some operating point. Steady state interaction measures may not always predict performance under dynamic control (McAvoy, 1981). In the non-linear case, inputs may affect each other by differing amounts in different ranges, or not at all. However, it significantly reduces the instability caused by interactions in this control scheme.

\section{EVALUATION OF THE MULTIPLE-DRUG SOFLC HEMODYNAMIC CONTROLLER}

The Multiple-Drug SOFLC (MSOFLC) with The Multivariable SOFLC (MSOFLC) with RGA decoupling and noise filter has been extensively tested on the physiological model in a simple closed-loop control scheme. The MAP and $\mathrm{CO}$ were simultaneously controlled using noradrenaline and dopamine. A noise signal with a power equivalent to $2 \%$ of the signal strength was added to the model outputs and a third-order polynomial filter was implemented, using a window of 20 data-points to perform adequate smoothing. The results of Fig. 6 show the simultaneous control of MAP and $\mathrm{CO}$ to their respective target values without and with the designed smoothing filter.
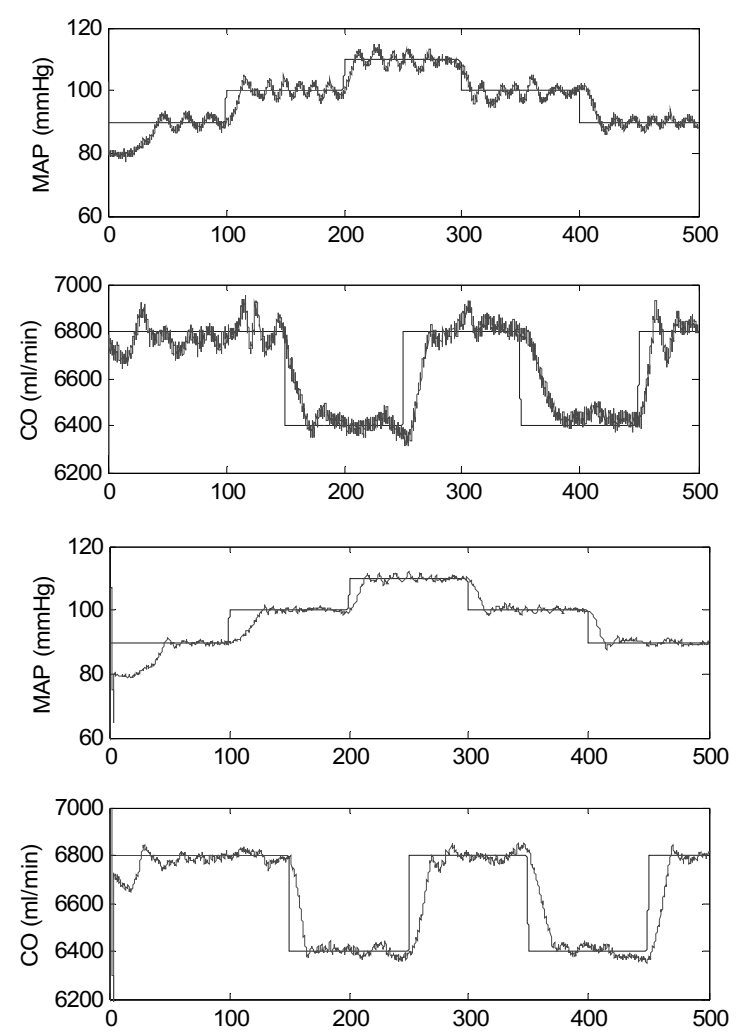

Fig. 6. MSOFCL control with RGA decoupling of MAP and $\mathrm{CO}$ with noradrenaline and dopamine respectively: Without smoothing filter (top panel) and with smoothing filter (bottom panel) 
The SOFLC was able to maintain control of MAP and CO within $10 \%$ of the target values. Polynomial filtering provides effective noise attenuation, however this adds a marked computational burden as least squares fitting must take place at each time step. The interactions effects among the controlled hemodynamic parameters have been successfully reduced using RGA decoupling, although this will not provide the same benefit at all ranges. The initial response time of the SOFLC can be improved using expert knowledge as it takes time for the controller to generate rules and for the control action to ramp up from zero.

\section{EVALUATION OF THE INTELLIGENT ADVISORY SYSTEM VIA THE PATIENT PHYSIOLOGICAL MODEL}

Patients with severe sepsis develop an increased microvascular permeability of the capillary barrier and interstitial edema. To simulate the capillary leak condition, the reflection coefficient $\sigma$ of the model was reduced by $30 \%$ causing a net shift of $500 \mathrm{ml}$ of fluid from the circulation to the interstitium. In addition the SVR was reduced and the contractility increased. As shown in Fig. 7, this caused the MAP and CVP to drop to $70 \mathrm{mmHg}$ and $5.2 \mathrm{mmHg}$ respectively. This in turn triggered the "low MAP" rule which started the infusion of a vasoconstrictor hence driving the MAP to $100 \mathrm{mmHg}$. This takes quite a long time because the SOFLC is starting from an empty rule-base. The CVP is still low, which triggers the "low CVP" rule hence initiating the infusion of a $250 \mathrm{ml}$ bolus of fluid at $12.5 \mathrm{ml} / \mathrm{min}$. Fluid administration caused, on the other hand MAP to increase over 120 , subsequently firing the "high MAP" rule. However, the way the expert's decision model is implemented here the "High MAP" will not start vasodilator therapy unless hypertension still persists after the infusion rate of the vasoconstrictor which is being administrated has been progressively reduced and stopped. As the amount of vasoconstrictor was reduced, the MAP decreased to 100 $\mathrm{mmHg}$. Note that this response is faster as the SOFLC rulebase has been filled from the first response.

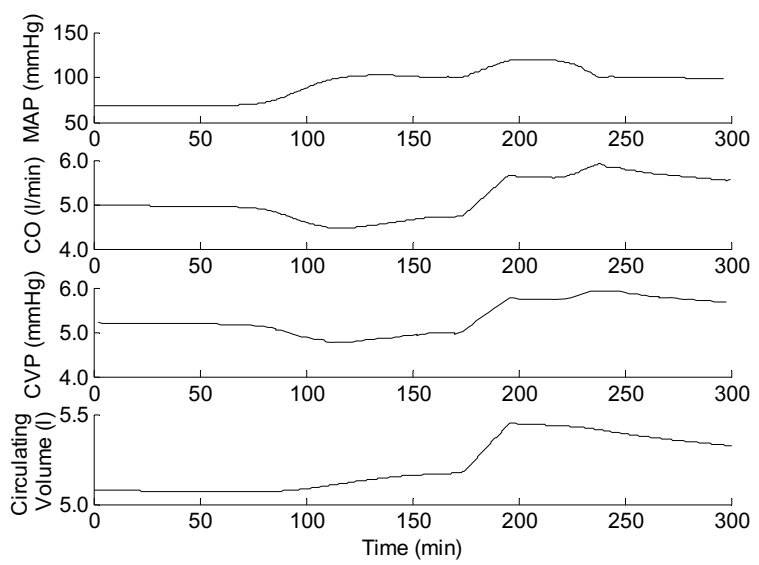

Fig.7. Simulation of a patient with capillary leak, vasodilation and increased contractility

In the scenario of Fig. 8 , the contractility $\left(K_{l}\right.$ and $\left.K_{r}\right)$ was reduced, causing a drop in cardiac output to $3.6 \mathrm{l} / \mathrm{min}$. As the model hadn't reached steady state at the beginning of the simulation, the pressure changes cause fluid shifts which are much slower than the other dynamics. This triggered the "low $\mathrm{CO}^{\prime}$ rule, and the $\mathrm{CO}$ was driven towards $5 \mathrm{l} / \mathrm{min}$ with dopamine.
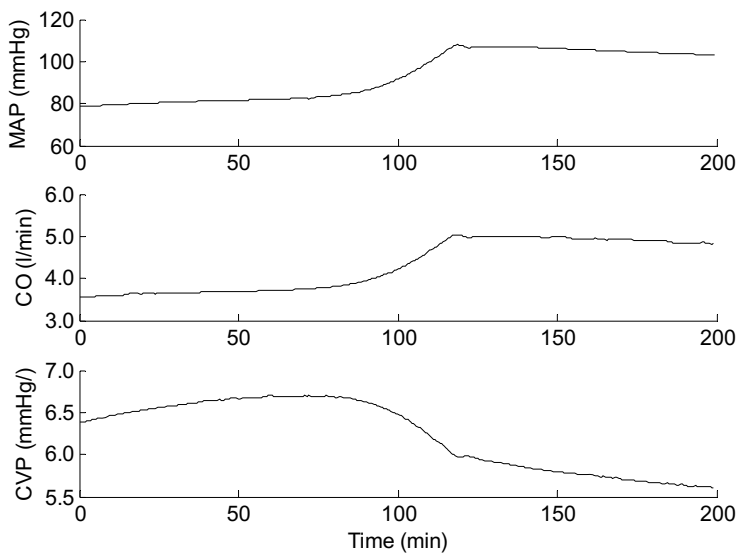

Fig. 8. Simulation of a patient with low $\mathrm{CO}$

To simulate hypertension, SVR was increased from its baseline value. As shown in Fig. 9, this has caused an increase in the MAP to $119 \mathrm{mmHg}$. The MAP setpoint was $95 \mathrm{mmHg}$ when the advisory system was started. This triggered the "high MAP" rule and the MAP is driven towards $95 \mathrm{mmHg}$ with a vasodilator. At 150 minutes, the MAP setpoint is reduced to $80 \mathrm{mmHg}$. This triggers the "high MAP" rule and the MAP is driven towards $80 \mathrm{mmHg}$ with a vasodilator.
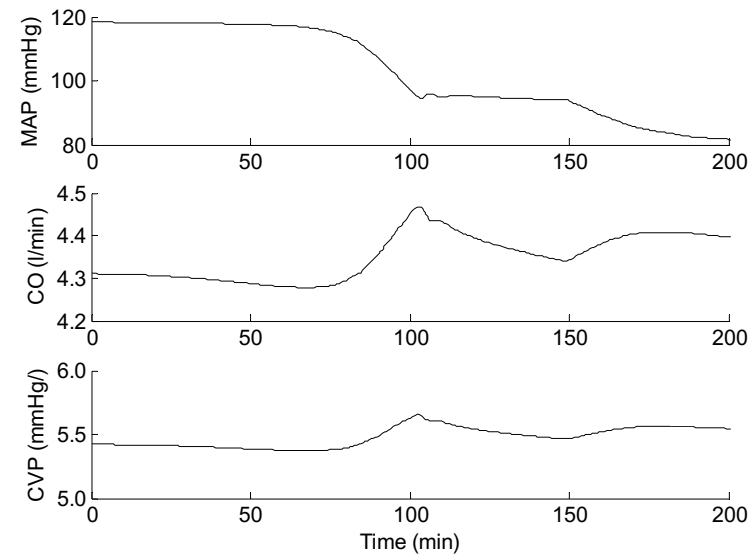

Fig. 9. Simulation of a patient with hypertension

In Fig. 10, the baseline parameters for $R_{s}, K_{l}, K_{r}$ and $\sigma$ have been changed from their nominal values to those simulating a septic patient. Fig. 10 starts when the MAP has dropped below $80 \mathrm{mmHg}$, and the first rule triggered was "low MAP" rule. The MAP is driven towards $100 \mathrm{mmHg}$ with a vasoconstrictor. The CVP is below $4 \mathrm{mmHg}$ therefore firing the "low CVP" rule and a $250 \mathrm{ml}$ bolus of fluid at $12.5 \mathrm{ml} / \mathrm{min}$ is given. The CVP is still low after this, triggering a second bolus. The MAP has risen to over $110 \mathrm{mmHg}$ by this stage triggering the "high MAP" rule. This drives the MAP back to $100 \mathrm{mmHg}$ by reducing the vasoconstrictor level. There is a third "low CVP" rule fluid bolus at $180 \mathrm{~min}$, followed by a "low CO" state. The $\mathrm{CO}$ is driven to $5 \mathrm{l} / \mathrm{min}$ with dopamine. 


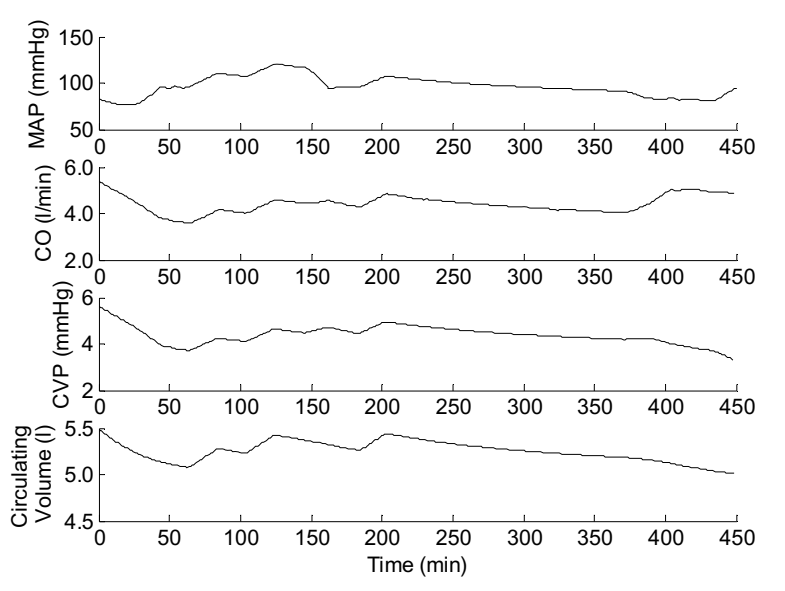

Fig. 10. Simulation of a septic patient

Finally, in Fig. 11, the contractility $\left(K_{l}, K_{r}\right)$ is reduced as well as the sensitivity of the model to dopamine. The model enters the "low CO" state and the advisory system reaches the maximum infusion of dopamine, then switches to the second drug choice for the "low CO" state.
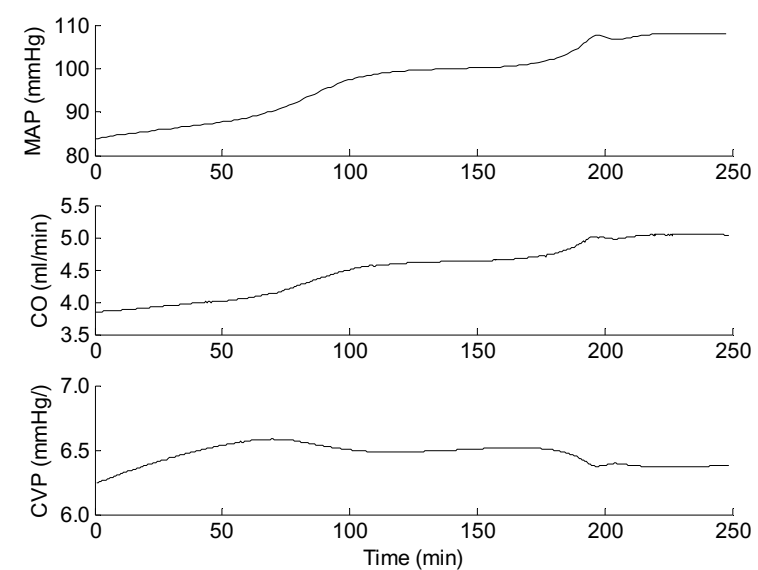

Fig. 11. Simulation of a patient with reduced contractility and drug sensitivity

\section{CONCLUSIONS}

Post-cardiopulmonary bypass (CPB) patients are susceptible to major hemodynamic instabilities as a result of the transient, multiple physiological derangements associated with $\mathrm{CPB}$ and the surgery itself. Sepsis/septic shock remain of the most frequently diagnosed conditions encountered in these critically ill patients. Supportive treatment of septic shock patients, parallel to reversing the cause of infection, includes fluid resuscitation and vasoactive medication to restore normal blood pressure and improve the circulatory performance. This control environment is well-defined and hierarchical which makes it suitable for a computerized implementation. The authors have developed a clinical advisory system for the management of post-cardiac septic patients. The hierarchical advisory system operating in closed-loop has been tested on a physiological model of the cardiovascular hemodynamics under a wide range of simulated pathological conditions characterizing post-CPB septic patients. In all the simulated patients scenarios the advisory system was able to trigger the adequate decision rule, initiate the right therapeutic action and control the hemodynamic parameters to the prescribed target values.

The proposed advisory has been designed with a sufficient degree of flexibility to allow future extensions to be made to enhance its functionality and performance. The system can be easily configured to accommodate a larger number of monitored variables and extended to accommodate alternative decision hierarchies to adhere with the clinician's workflow and/or preference in the management of post-CPB patients.

\section{Acknowledgements}

The authors gratefully acknowledge the financial support from the United Kingdom Engineering and Physical Sciences Research Council (EPSRC) under Grants GR/S94636/1 and EP/FO2889X/1

\section{REFERENCES}

Dellinger R.P. (2003). Cardiovascular Management of Septic Shock, Crit Care Med, 31(3), 946-955.

Bridges E.J. and S. Dukes (2005). Cardiovascular Aspects of Septic Shock: pathophysiology, monitoring, and treatment, Crit. Care Nurse, 25(2), 14-42.

Parrillo J.E. (1989). The Cardiovascular Pathophysiology of Sepsis, Annual Rev Med., 40, 469-485.

Astiz M.E. and E. C. Rackow (1998). Septic Shock, Lancet, 351(9114), 1501-5.

Grocott M.P.W. and Mythen M.G., Fluid Administration in Septic Shock, Sepsis 2001, 2, 111-124.

Randall J.E., A Cardiovascular System Model in: Microcomputers and Physiological Simulation, Raven Press, 1986.

Mason D.G. (1989). Comprehensive Circulatory Management of Seriously Ill patients with a Closed-loop System, PhD Thesis for Dept of Electrical and Electronic Engineering, University of Melbourne.

Xie S.L., R.K. Reed, B.D. Bowen and J.L. Bert (1995). A Model of Human Microvascular Exchange, Microvascular Research, 49(2), 141-62.

Gyenge C.C., B.D. Bowen, R.K. Reed and J.L. Bert (1999). Transport of Fluid and Solutes in the Body I. Formulation of a Mathematical Model, Am. J. Physiol. Heart Circ. Physiol., 277, H1215-H1227.

Klabunde R.E (2004). Cardiovascular Physiology Concepts, Lippincott Williams and Winkins.

Hamada Y., K. Kawachi, N. Tsunooka, Y. Nakamura, S. Takano and H. Imagawa (2004). Capillary leakage in cardiac surgery with cardiopulmonary bypass, Asian Cardiovasc Thorac. Ann., 12(3), 193-197.

Ross J.J., M. Mahfouf, M. Denaï and O.K. King (2006). Modelling Decision-Making Strategies in Supporting the Shocked Patient, British Journal of Anesthesia, 97, 432.

Procyk T.J. and E.H. Mamdani (1979). A Linguistic SelfOrganizing Process Controller, Automatica, 15, 15-30.

Bristol E. (1966). On a New Measure of Interaction for Multivariable Process Control, IEEE Transactions on Automatic Control, 11, 133-134.

McAvoy T.J. (1981). Connection between Relative Gain and Control Loop Stability and Design, AIChE Journal, 27, 613-619. 